\title{
The Cortical Rhythms of Chronic Back Pain
}

\author{
Marwan N. Baliki, ${ }^{1}$ Alex T. Baria, ${ }^{1}$ and A. Vania Apkarian ${ }^{1,2,3,4}$ \\ Departments of ${ }^{1}$ Physiology, ${ }^{2}$ Anesthesia, and ${ }^{3}$ Surgery, and ${ }^{4}$ Rehabilitation Institute of Chicago, Feinberg School of Medicine, Northwestern University, \\ Chicago, Illinois 60610
}

Chronic pain is maladaptive and influences brain function and behavior by altering the flow and integration of information across brain regions. Here we use a power spectral analysis to investigate impact of presence of chronic pain on brain oscillatory activity in humans. We examine changes in BOLD fluctuations, across different frequencies, in chronic back pain (CBP) patients $(n=15)$ as compared to healthy controls $(n=15)$ during resting-state fMRI. While healthy subjects exhibited a specific, frequency band-dependent, large-scale neural organization, patients showed increased high-frequency BOLD oscillations $(0.12-0.20 \mathrm{~Hz})$ circumscribed mainly to medial prefrontal cortex (mPFC) and parts of the default mode network. In the patients a correlation analysis related the mPFC aberrant BOLD high-frequency dynamics to altered functional connectivity to pain signaling/modulating brain regions, thus linking BOLD frequency changes to function. We also found that increased frequency fluctuations within the mPFC were temporally synchronous with spontaneous pain changes in patients during a pain-rating task. These observations provide novel insights about the nature of CBP, identifying how it disturbs the resting brain, and link high-frequency BOLD oscillations to perception.

\section{Introduction}

Chronic pain is complex in nature and cannot be localized to a unitary set of regions in the brain; rather, it emerges from the flow and integration of information between multiple brain regions (Cauda et al., 2009b; Apkarian et al., 2011). In addition to the brain's normal resting activity, the brain of chronic pain patients is continuously processing spontaneous background pain, which interacts with and modulates endogenous brain networks (e.g., attention/salience/emotional/reward and sensory networks). Thus resting-state $\mathrm{fMRI}$ provides a suitable framework to examine the brain mechanisms associated with the "natural state" of chronic pain (i.e., spontaneous pain in the absence of experimental intervention). Evidence for the impact of chronic pain on the default mode network $(\mathrm{DMN})$, a set of brain regions active at rest and involved in self-referential orientation and monitoring (Fox et al., 2005), was first shown in chronic back pain (CBP) patients (Baliki et al., 2008). More recently, multiple observations of abnormal connectivity within various resting-state networks have been reported for different chronic pain conditions (Cauda et al., 2009a; Napadow et al., 2010; Tagliazucchi et al., 2010).

Recent studies have demonstrated that investigating brain properties as a function of frequency of BOLD oscillations reveals novel anatomical/functional organization and identifies the impact of extrinsic tasks on the intrinsic properties of the brain (Salvador et al., 2008; Baria et al., 2011). The advantage of this approach, as opposed

\footnotetext{
Received April 20, 2011; revised Aug. 3, 2011; accepted Aug. 9, 2011.

Author contributions: M.N.B. and A.V.A. designed research; M.N.B. and A.T.B. performed research; M.N.B. and A.T.B. analyzed data; M.N.B. and A.V.A. wrote the paper.

The study was funded by NIH-NINDS Grant NS35115. M.N.B. was funded by an anonymous foundation. We thank all patients and healthy volunteers that participated in the study, and M. A. Farmer for reading and editing the paper.

Correspondence should be addressed to A. Vania Apkarian at the above address. E-mail: a-apkarian@ northwestern.edu.

DOI:10.1523/JNEUROSCI.1984-11.2011

Copyright $\odot 2011$ the authors $\quad 0270-6474 / 11 / 3113981-10 \$ 15.00 / 0$
}

to correlation-based analyses, rests in its ability to identify oscillatory dynamics of the BOLD signal along a wider range of frequencies. Thus investigating changes in the brain's BOLD oscillatory dynamics may provide us with novel insight regarding the neural mechanisms underlying chronic pain. For instance, two recent studies examined changes in power for BOLD oscillations during restingstate fMRI (Cauda et al., 2010; Malinen et al., 2010). Both studies reported increased high-frequency oscillations $(>0.12 \mathrm{~Hz})$ within certain brain regions in patients compared to controls. Although both studies provided novel observations on chronic pain processing, they were confounded by two limitations: (1) they assessed changes in spectral power on localized predefined brain networks, and (2) neither study was able to directly relate the observed frequency-dependent changes in fluctuations with behavioral outcomes, namely spontaneous pain. Both of these issues are addressed in the current study.

Here we use a whole-brain, full-bandwidth, power spectral analysis to investigate changes in the oscillatory dynamics of BOLD across different frequencies in CBP patients $(n=15)$, compared to age- and gender-matched healthy controls, during resting-state fMRI. Our aims are (1) to characterize and spatially localize altered oscillatory dynamics along the whole frequency band of the BOLD signal in CBP and (2) to relate these changes to functional connectivity alterations within resting-state networks. We also scan a subgroup of the CBP population $(n=13)$ while they rate their online changes in spontaneous pain in the absence of external stimulation. The purpose of this experiment is to delineate the temporal relationship between changes in oscillatory properties of the BOLD and spontaneous CBP, thus linking intrinsic function to extrinsic behavior.

\section{Materials and Methods}

Participants. Fifteen healthy subjects ( 10 males, 5 females; age: mean $=$ $51.87, \mathrm{SD}=8.26$ years $)$ and $15 \mathrm{CBP}$ patients $(10$ males, 5 females; age: mean $=49.85, \mathrm{SD}=9.90$ years) participated in this study. All partici- 
Table 1. Demographics and clinical data for CBP patients that participated in the study

\begin{tabular}{|c|c|c|c|c|c|c|c|c|c|c|}
\hline Code & Gender & Age (years) & Duration (years) & $\mathrm{RC}$ & $\operatorname{VAS}(0-100)$ & MQS & BDI & MPQ sensory & MPQ affective & Reported origin/cause \\
\hline 01 & $\mathrm{~F}$ & 51 & 15 & Yes & 80 & 34.8 & 15 & 10 & 2 & Spontaneous \\
\hline 02 & M & 62 & 1 & Yes & 87 & 5.7 & 1 & 8 & 0 & Spontaneous \\
\hline 03 & $\mathrm{~F}$ & 49 & 12 & Yes & 84 & 44.9 & 12 & 6 & 5 & Injury \\
\hline 04 & $\mathrm{~F}$ & 62 & 13 & Yes & 84 & 17.5 & 13 & 12 & 6 & Spontaneous \\
\hline 05 & $\mathrm{~F}$ & 60 & 8 & Yes & 100 & 20.9 & 8 & 13 & 3 & Spontaneous \\
\hline 06 & $\mathrm{~F}$ & 54 & 6 & Yes & 75 & 24 & 6 & 7 & 2 & Spontaneous \\
\hline 07 & $M$ & 50 & 4 & Yes & 69 & 12.5 & 4 & 9 & 0 & Spontaneous \\
\hline 08 & M & 54 & 4 & Yes & 62 & 8.9 & 4 & 12 & 5 & Spontaneous \\
\hline 09 & M & 62 & 3 & Yes & 54 & 18.1 & 3 & 17 & 1 & Injury \\
\hline 10 & $M$ & 46 & 13 & Yes & 59 & 53.8 & 13 & 5 & 1 & Spontaneous \\
\hline 11 & M & 49 & 4 & Yes & 63 & 11.3 & 4 & 10 & 7 & Spontaneous \\
\hline 12 & M & 52 & 6 & Yes & 57 & 6.9 & 6 & 9 & 5 & Spontaneous \\
\hline 13 & $M$ & 54 & 4 & No & 62 & 27.3 & 4 & 13 & 3 & Surgery/injury \\
\hline 14 & $M$ & 32 & 5 & Yes & 78 & 25.2 & 5 & 7 & 7 & Spontaneous \\
\hline 15 & $M$ & 41 & 3 & Yes & 30 & 11.7 & 3 & 15 & 9 & Spontaneous \\
\hline
\end{tabular}

VAS, Visual analog scale; RC, radicular component. Resting-state scans were acquired for all patients. Pain and visual rating scans were acquired for patients $01-13$.

pants were right-handed, and gave informed consent to procedures approved by Northwestern University Institutional Review Board committee. All patients, recruited by newspaper or Internet ads in Chicago area, were clinically diagnosed with CBP by a clinician, had back pain of intensity $>20 / 100$ and duration $>1$ year, and had to fulfill a specific list of exclusion criteria. Subjects were excluded if they reported other chronic painful conditions, systemic disease, history of head injury or coma, psychiatric diseases, or more than mild to moderate depression (BDI score > 19), as defined by Beck's Depression Inventory (BDI) (Beck and Steer, 1993).

Pain and depression parameters. CBP patients completed the shortform of the McGill pain questionnaire (SF-MPQ) (Melzack, 1987). The main component of the SF-MPQ consists of 12 sensory and 4 affective descriptors; it also includes a visual analog scale $(0=$ no pain, $100=$ maximum imaginable pain) and pain duration. Depression scores for all subjects that participated in the study were assessed using BDI. All questioners were given $1 \mathrm{~h}$ before brain scanning. Clinical and pain characteristics for CBP are listed in Table 1.

Medication. Drug consumption for 10 CBP patients was limited to nonsteroidal anti-inflammatory drugs and/or acetaminophen (e.g., ibuprofen, Motrin, Excedrin, and Tylenol). Three subjects were additionally on gabapentin (Neurontin) or pregabalin (Lyrica). Two subjects were on opiates (Vicodin). Drug consumption was quantified using the Medication Quantification Scale (MQS) (Harden et al., 2005), which reduces drugs used for different durations and doses to a single scalar. The MQS was used as a covariate of no interest to control for drug usage. However, inclusion of this covariate did not result in important changes in outcomes.

fMRI data and acquisition. $\mathrm{AMRI}$ data were acquired with a $3 \mathrm{~T}$ Siemens Trio whole-body scanner with echo-planar imaging capability using the standard eight-channel radio-frequency head coil. The anatomical and fMRI scans were collected during a single brain imaging session with scan sequence randomized across subjects.

Resting-state scans (300 volumes, $12 \mathrm{~min}$ ) were acquired for all healthy $(n=15)$ and CBP $(n=15)$ subjects. All participants had no task but were instructed to stay alert and keep their eyes open for the duration of the scan. Images were obtained with the following parameters: Multislice $\mathrm{T} 2{ }^{\star}$-weighted echo-planar images were repetition time $(\mathrm{TR})=2.5 \mathrm{~s}$, echo time $(\mathrm{TE})=30 \mathrm{~ms}$, flip angle $=90^{\circ}, \mathrm{FOV}=256 \mathrm{~mm}$, slice thickness $=3 \mathrm{~mm}$, in-plane resolution $=64 \times 64$. The 40 slices covered the whole brain from the cerebellum to the vertex.

Pain and visual rating scans ( 240 volumes, $10 \mathrm{~min}$ ) were acquired in the same session but in separate scans, for 13 of the 15 CBP patients. Before scanning, these patients underwent an initial training in which they learned to use the finger-span device, comprised of a potentiometer the voltage of which was digitized and time stamped in reference to fMRI image acquisition and connected to a computer providing visual feedback of the ratings. During the pain rating scans, subjects were instructed to rate fluctuations of the intensity of their spontaneous back pain using the finger-span device on a scale of $0-100$, where 0 is no pain and 100 is the maximum imaginable pain. Subjects were provided with an online visual feedback of their ratings. During the visual rating scan, subjects were instructed to rate the length of the bar projected on a screen using the fingerspan device. Unbeknown to the subjects, the variability of the bar was derived from their pain rating scans. Thus, this task serves as a control for task-related activations such as attention, anticipation, and visual-motor performance. Functional images for the pain and visual rating scans were obtained with the following parameters: multislice $\mathrm{T} 2^{\star}$-weighted echoplanar images were $\mathrm{TR}=2.5 \mathrm{~s}$, TE $=30 \mathrm{~ms}, \mathrm{FOV}=270 \mathrm{~mm}$, flip angle $=$ $90^{\circ}$, slice thickness $=3 \mathrm{~mm}$, in-plane resolution $=64 \times 64$. The 36 slices covered the whole brain from the cerebellum to the vertex.

In addition to the functional scans, a T1-weighted anatomical MRI image was also acquired for each subject using the following parameters: $\mathrm{TR}=2.1 \mathrm{~s}, \mathrm{TE}=4.38 \mathrm{~ms}$, flip angle $=8^{\circ}, \mathrm{FOV}=220 \mathrm{~mm}$, slice thickness $=1 \mathrm{~mm}$, in-plane resolution $=0.861 \times 0.861 \mathrm{~mm}^{2}$, and number of sagittal slices $=160$

fMRI image preprocessing. The preprocessing of each subject's time series of fMRI volumes was performed using the FMRIB Expert Analysis Tool (FEAT, Smith et al., 2004, www.fmrib.ox.ac.uk/fsl) and encompassed the following: skull extraction using BET, slice time correction, motion correction, spatial smoothing using a Gaussian kernel of fullwidth at half-maximum $5 \mathrm{~mm}$, and high-pass temporal filtering (150 s). Several sources of noise, which may contribute to non-neuronal fluctuations, were removed from the data through linear regression. These included the six parameters obtained by rigid body correction of head motion, the global BOLD signal averaged over all voxels of the brain, signal from a ventricular region of interest, and signal from a region centered in the white matter.

Spectral power analysis. Spectral analysis was performed using custom Matlab (The MathWorks, 2009) routines, and closely follows procedures outlined by Baria et al. (2011). Frequency power of the BOLD signal was determined voxelwise using Welch's method and normalized by dividing by total power. The average power of each frequency band (low frequency: 0.01-0.05 Hz, middle frequency: $0.05-0.12 \mathrm{~Hz}$, and high frequency: $0.12-$ $0.20 \mathrm{~Hz}$ ) was calculated at each voxel and made up three different maps for each subject. Individual subject maps were transformed into standard space using FLIRT (Jenkinson and Smith, 2001) and multiplied by a standard gray matter mask. Group mean spectral distribution maps were generated by averaging maps across subjects, for each group separately. Individual spectral distribution maps for each frequency band were submitted separately to a two-sided unpaired $t$ test with age and gender as covariates of no interest. Statistical differences between the two groups were computed using a random-effects analysis $(z$-score $>3.0$, cluster threshold $p<0.01$, clusterbased corrected for multiple comparisons).

Region of interest and BOLD analysis. The regions of interest (ROIs) were fixed-size, 6-mm-diameter spheres, centered at peak coordinates defined from brain power spectral density and brain correlation (see below) analyses. The medial prefrontal cortex (mPFC) ROI was defined from the spectral power contrast map for the high-frequency band with 
A
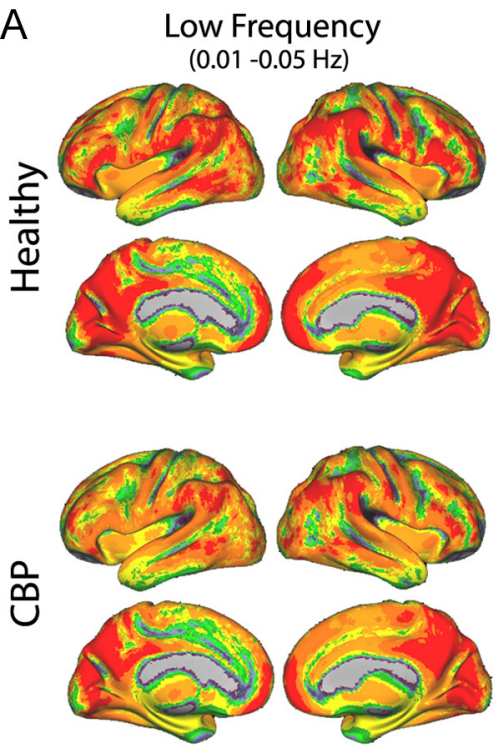

10

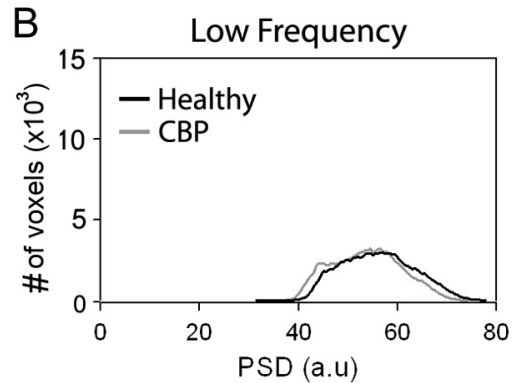

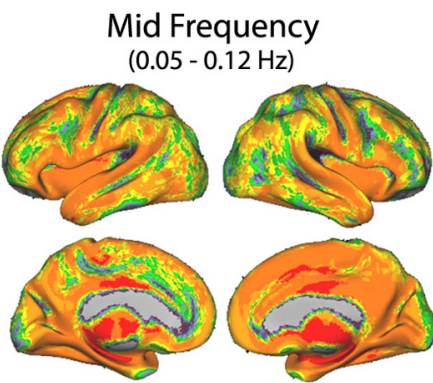
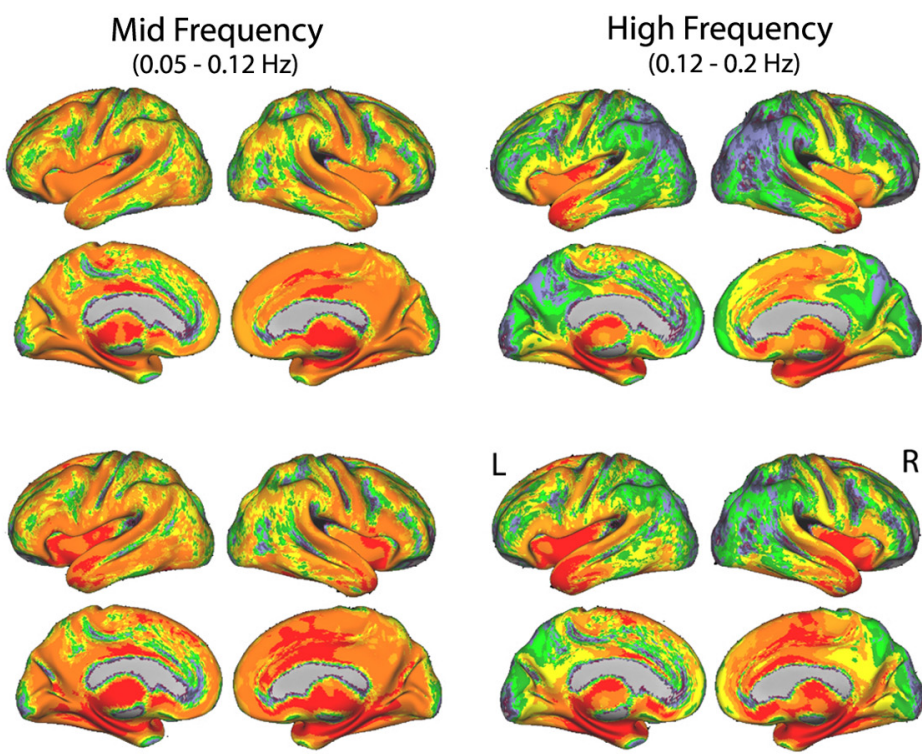

10

45

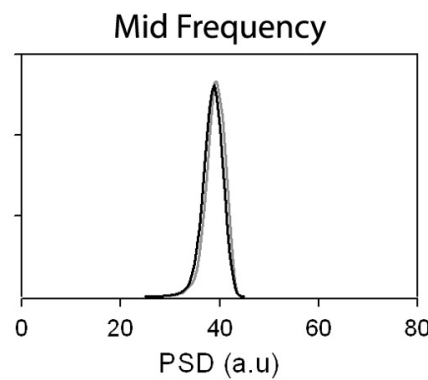

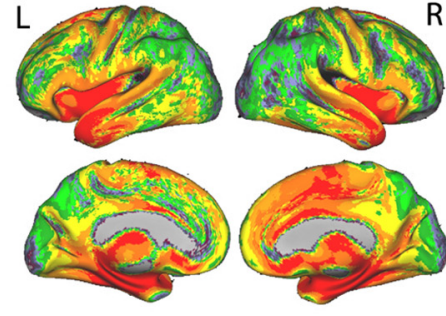

$10 \square \square=30$

High Frequency

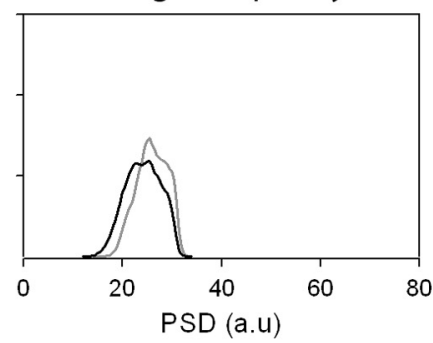

Figure 1. Spatial distribution of spectral power for BOLD oscillations in healthy subjects and CBP during resting-state fMRI. $A$, Brain maps show spatial distribution of the group average spectral power for the three frequency bands (low: $0.01-0.05 \mathrm{~Hz}$, middle: $0.05-0.12 \mathrm{~Hz}$, and high: $0.12-0.20 \mathrm{~Hz}$ ) for CBP patients $(n=15)$ and healthy controls $(n=15)$. In the control group, the lowest frequency exhibited highest power, and was localized mainly to prefrontal, parietal, and occipital cortices. The mid- and high-frequency bands consecutively exhibited less power, and localized more within cingulate, insular, and temporal cortices, in addition to subcortical structures. The CBP group generally shows a similar pattern of spatial distribution of spectral power to the healthy group, with increases in the high-frequency power in parts of the medial frontal and insular regions and mid-frequency power in the cingulate cortex. B, Group average histograms of power spectral density (PSD) for BOLD oscillations for the three frequency bands in healthy subjects (black trace) and CBP (gray trace).

coordinates: $x=-6, y=58, z=0$. The anterior cingulate cortex (ACC), insula (INS), and secondary somatosensory cortex (S2) ROIs were defined from the correlation analysis contrast maps with the following coordinates: ACC: $x=6, y=8, z=32$; INS: $x=42, y=0, z=-4$; S2: $x=60, y=-32, z=30$. To extract the BOLD signal for each ROI and subject, the ROIs were reverse normalized and projected back into individual brain space, and BOLD signal for the total trial duration was obtained by averaging the raw data for all voxels across a given ROI. BOLD time course was measured by calculating percentage BOLD change (deviation from the mean for voxels within the ROI).

Functional connectivity analysis. Brain mPFC correlation maps were identified using a well validated method (Fox et al., 2005; Baliki et al., 2008). Correlation maps were produced for the resting-state functional scans by first extracting the BOLD time course from the mPFC ROI and then computing correlation coefficient between its time course and the time variability of all other brain voxels. Correlation coefficients were converted to a normal distribution using the Fisher's $z$-transform. These values were then converted to $z$-scores (i.e., normalized correlation values) by dividing by the square root of the variance, estimated as $1 / \sqrt{\mathrm{df}-3}$, where $\mathrm{df}$ represents the degrees of freedom in our measurement ( 300 data points). Because the BOLD time courses of consecutive samples are not statistically independent, the degrees of freedom were corrected by a factor of 2.86 , in accordance to Bartlett theory (Jenkins and Watts, 1968), resulting in 300/2.86 = 104 degrees of freedom. A two-sided unpaired $t$ test was used to compute significant differences in correlations (Fisher's $z$-transformed values) between the two groups using a random-effects analysis $(z$-score $>3.0$, cluster threshold $p<0.01$, cluster-based corrected for multiple comparisons).

Spectral power and spontaneous pain ratings. The spectrogram for the mPFC BOLD time series was generated using the custom Matlab (The MathWorks, 2009) signal-processing toolbox. The spectrogram was computed using short-time Fourier transform of the input signal with $95 \%$ window overlap. The averaged spectral power for the low- $(0.01-$ $0.05 \mathrm{~Hz})$, mid- $(0.05-0.12 \mathrm{~Hz})$, and high- $(0.12-0.25)$ frequency bands were derived from the spectrogram by averaging across all frequencies for a given band and normalizing with respect to its mean. Correlation between the averaged spectral power of each band and the pain or visual rating was computed using a Pearson correlation after convolving the rating with homodynamic response function and temporal resampling to match the time course of the spectrogram.

Surface-based mapping. Surface-based mapping (Fig. 1) was constructed using the PALS (population-average, landmark- and surfacebased) average fiducial surface from 12 individual subjects as the atlas target (Van Essen, 2005).

\section{Results}

CBP is coupled with increased high-frequency BOLD oscillations in the brain

We first assessed whole-brain full-bandwidth spectral properties of BOLD oscillations for three frequency bands (low: 0.01-0.05 
$\mathrm{Hz}$, middle: $0.05-0.12 \mathrm{~Hz}$, and high: $0.12-0.20 \mathrm{~Hz}$ ) for healthy subjects and CBP patients during resting-state fMRI. The three frequency bands were defined based on a previous report (Malinen et al., 2010) that showed frequency-dependent changes for BOLD oscillations between healthy subjects and chronic pain patients, as well as on results of Baria et al. (2011). In healthy subjects, the spatial distribution of power for the three frequency bands showed segregation, which corroborated our previous findings (Baria et al., 2011) and was consistent with previous reports (Mitra et al., 1997; Fukunaga et al., 2006; Duff et al., 2008; Salvador et al., 2008). Low-frequency power was strongest in lateral parietal regions and along the midline, especially the $\mathrm{mPFC}$, posterior cingulate cortex (PCC), and the visual regions. In contrast, mid-frequency power was primarily localized to middle portions of the cingulate (ACC), bilateral insula (INS), and subcortical nuclei including the basal ganglia and thalamus. High-frequency power exhibited a similar spatial distribution and was mainly located in the ACC, INS, and subcortical regions, in addition to temporal poles and hippocampal formation. Visually, CBP patients exhibited similar anatomical segregations across the three frequency bands, albeit an increase in spatial representation and increased power for the mid- and high-frequency bands in the $\mathrm{ACC}$ and insula and a decrease of low-frequency power in the $\mathrm{mPFC}$ (Fig. 1A).

For both groups, spectral power was strongest for the lowfrequency band (i.e., frequencies below $0.05 \mathrm{~Hz}$ ) and reduced over the mid- and high-frequency bands. Figure $1 B$ show groupaveraged histograms of power distributions for the three frequency bands for all voxels in the brain in healthy subjects and CBP patients. The low-frequency band showed the highest power and widest distribution (healthy: $56.01 \pm 7.31$, mean $\pm \mathrm{SD}$; range: $31.71-77.75$; CBP: $53.67 \pm 7.00$; range: $32.79-73.86)$. The mid-frequency band showed lower power and the narrowest distribution (healthy: $38.66 \pm 1.99$; range: $24.10-44.68$; CBP: $39.11 \pm 1.97$; range: $24.16-44.16$ ). The high-frequency band exhibited the lowest power and intermediate width (healthy: $24.20 \pm 3.55$; range: $12.25-33.55$; CBP: $25.98 \pm 3.70$; range: $14.04-33.24)$.

\section{Increased high-frequency BOLD oscillations are localized to the mPFC in CBP}

To localize the brain regions that exhibited changes in spectral power between healthy subjects and CBP patients, we performed a whole-brain voxelwise contrast analysis separately for the three frequency bands, after $z$-transforming individual brain- and band-specific power distributions (unpaired two-sided $t$ test, random-effects analysis, $z$-score $>3.0$, cluster threshold $p<$ 0.01 , cluster-based corrected for multiple comparisons). Results are shown in Figure 2A. Only the high-frequency band showed significant differences in spectral power between the two groups after correcting for multiple comparisons. CBP patients showed increased power for high-frequency BOLD oscillations primarily in the mPFC (cluster size $=1595$ voxels; peak coordinates: $x=$ $-6, y=58, z=0$; peak $z$-value $=4.98$ ). Other regions included the PCC cluster size $=450$ voxels; peak coordinates: $x=12, y=$ $-60, z=22$; peak $z$-value $=3.91$ ), left lateral parietal cortex (LP; cluster size $=512$ voxels; peak coordinates: $x=-32, y=-62$, $z=38$; peak $z$-value $=3.79$ ), and right lateral parietal cortex (cluster size $=507$ voxels; peak coordinates: $x=44, y=-56, z=$ 48 ; peak $z$-value $=3.50$ ). Note that the four brain regions ( $\mathrm{mPFC}$, PCC, and right and left LP) that show increased power in highfrequency BOLD oscillations are components of the DMN, indi- cating that the $\mathrm{DMN}$ is the primary resting-state network modulated in frequency by CBP.

Figure $2 B$ shows the corresponding individual $\mathrm{mPFC} B O L D$ time course from healthy subjects and CBP patients. The BOLD signal was extracted from a 6-mm-diameter ROI region centered on the peak coordinates. The temporal patterns differ upon visual inspection. While the mPFC BOLD signal from healthy subjects display slow rhythmic fluctuations, those from the CBP patients show increased high-frequency jitter. Figure $2 C$ shows the individual power spectra for the MPFC BOLD signal in healthy subjects (left panel, black traces) and CBP patients (right panel, gray traces). Consistent with time series observations, the power spectra in CBP patients show more power for higher frequencies. When we statistically compared the power for the three bands between the two groups, we observed a significant increase in power for the highfrequency band in CBP patients compared to healthy subjects (healthy: $14.17 \pm 1.87$; CBP: $21.61 \pm 6.04$; two-sided unpaired $t$ test: $t=-4.49, p<0.01)$. This increase in high-frequency power in CBP patients was accompanied by a decrease in power for the lowfrequency band (healthy: $74.39 \pm 2.99$; CBP: $70.05 \pm 4.74$; twosided unpaired $t$ test: $t=3.45, p<0.01$ ). There were no differences in the power for the mid-frequency band between the two groups (healthy: $33.83 \pm 1.09$; CBP: $33.45 \pm 0.98$; two-sided unpaired $t$ test: $t=0.96, p=0.85$ ) (Fig. 2D).

\section{mPFC frequency changes is related to functional connectivity in $\mathrm{CBP}$}

Here we examine the relationship between frequency changes in the $\mathrm{mPFC}$ in CBP and its intrinsic connectivity to the rest of the brain during resting-state $\mathrm{AMRI}$. First we generated whole-brain connectivity maps for the mPFC by computing correlation coefficients between its BOLD time course and the time variability of all other brain voxels (see Materials and Methods for details). After registering the individual connectivity maps to standard space, we submit them to an unpaired $t$ test analysis (randomeffects analysis, $z$-score $>3.0$, cluster threshold $p<0.01$ corrected for multiple comparisons). Results are shown in Figure $3 A$. The $\mathrm{mPFC}$ exhibited increased correlation to ACC $(x=6, y=8$, $z=32$ ), right INS $(x=42, y=0, z=-4)$, and secondary somatosensory cortex (S2; $x=60, y=-32, z=30)$ in CBP compared to controls. It is worthy to note that all these regions are known to receive nociceptive input from the periphery and play seminal roles in pain perception and modulation (Price, 2000; Apkarian et al., 2005).

Next we investigated the relationship between differences in correlation and increased spectral power observed in the mPFC signal. Figure $3 B$ shows the relationship between the strength of mPFC-ACC BOLD time course correlation $(6 \mathrm{~mm}$ ROI in each area) and its spectral power for the high-frequency band for the mPFC. As seen above for whole-brain contrast, the mPFC ROI showed altered correlation to the ACC ROI (measured by normalized correlation values, $z$-scores) in CBP compared to healthy subjects (healthy: $-1.28 \pm 1.28$; CBP: $0.68 \pm 1.11$; two-sided unpaired $t$ test: $t=-4.25, p<0.01)$. More importantly, this altered correlation showed a significant positive relationship with spectral power for the high-frequency band in CBP but not in healthy subjects (healthy: $r=-0.05, p=0.85$; CBP: $r=0.83, p<$ $0.01)$. Furthermore, the mPFC-INS correlation was also significantly altered in CBP (healthy: $-1.20 \pm 1.12$; CBP: $0.98 \pm 1.31$; two-sided unpaired $t$ test: $t=-4.73, p<0.01)$ and also was related to increased spectral power for the high-frequency band in the $\mathrm{mPFC}$ BOLD time course in CBP (healthy: $r=0.24, p=$ 0.38; CBP: $r=0.82, p<0.01$ ) (Fig. $3 C$ ). Similar to the mPFC- 


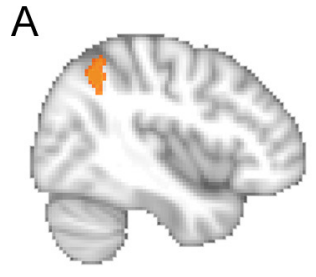

$x=40$

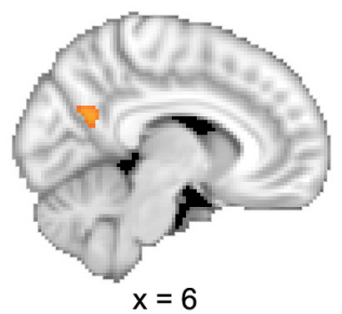

$x=6$

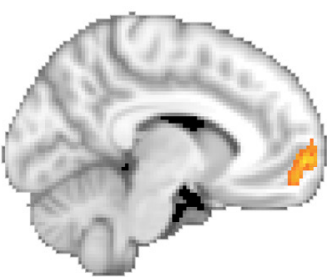

$x=-2$

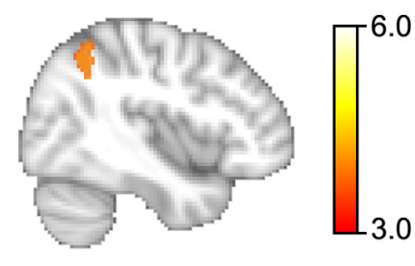

$x=-40$
B

$$
\text { (n) }
$$
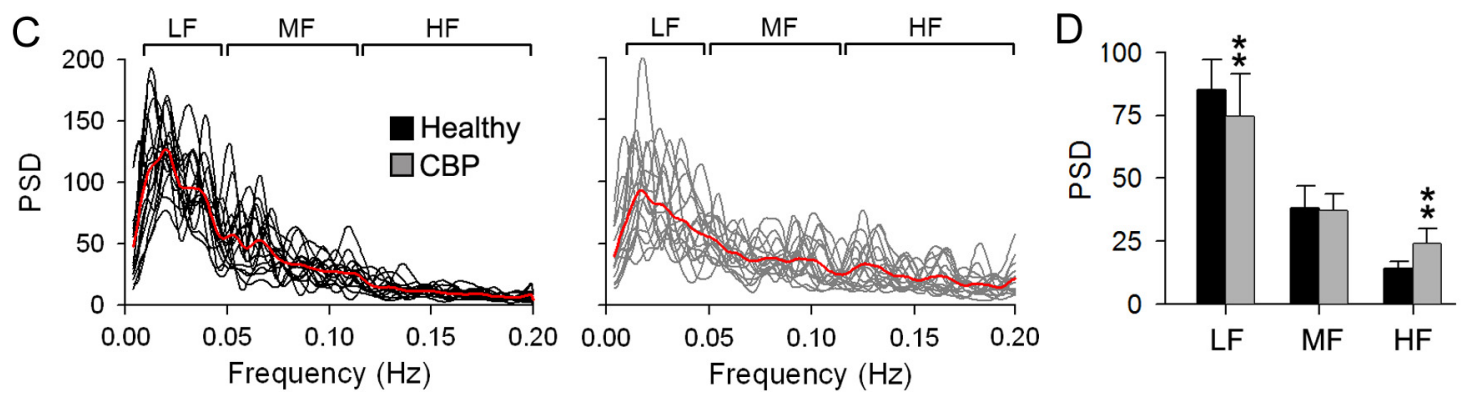

Figure 2. Regional differences in power spectral density (PSD) between healthy subjects and CBP during resting-state fMRI. $A$, Whole-brain voxelwise differences in power for the high-frequency band between CBP and healthy subjects. Brain areas in red-yellow depict statistically significantly higher power in CBP compared to controls (unpaired $t$ test, random-effects model, $z$-score $>3.0$, cluster $p<0.01$, corrected for multiple comparisons), localized mainly to the mPFC, PCC, and bilateral LP. No significant differences were detected for the mid- and low-frequency bands. Brain images are in standard MNI space, and coordinates are in millimeters. $\boldsymbol{B}$, Individual BOLD time courses from the mPFC for healthy subjects (left) and CBP (right). $C$, Individual power spectra for the mPFC BOLD time courses superimposed separately for healthy subjects (black traces) and (BP (gray traces). Red traces represent group averages. D, Bar graphs show the mean \pm SD spectral power from the mPFC time courses for the three frequency bands in healthy subjects (black bars) and CBP (gray bars). CBP exhibited significant decrease in the PSD for the low-frequency (LF) band, no change for the mid-frequency (MF) band, and an increase in the high-frequency (HF) band. [Compare to Malinen et al. (2010), their Figs. 2-4.]

ACC and mPFC-INS changes, mPFC-S2 exhibited significantly altered correlation in CBP (healthy: $-1.41 \pm 0.73$; CBP: $0.07 \pm$ 0.05 ; two-sided unpaired $t$ test: $t=-4.34, p<0.01$ ) and also was related to increased spectral power for the high-frequency band in the mPFC BOLD time course in CBP (healthy: $r=0.19, p=$ 0.48 ; CBP: $r=0.71, p<0.01$ ) (Fig. 3D). These results show that the increase in high-frequency BOLD oscillations in mPFC in CBP are accompanied by increased functional connectivity (more positive correlations) with multiple brain regions involved in nociceptive processing, and this enhanced connectivity is due to enhanced power in the high-frequency BOLD.

It is worthy to note that all correlation analyses were performed after regressing the global BOLD signal (see Materials and Methods). However, regressing out the global signal has been shown to introduce anti-correlations that might be artifactual (Van Dijk et al., 2010). To corroborate our findings, we performed the same analysis without regressing out the global BOLD signal. The mPFC connectivity contrast ( $\mathrm{CBP}>$ healthy) produced similar results with the MPFC exhibiting increased correlation to ACC, INS, and S2 (contrast maps for the two analyses showed $94.7 \%$ overlap, data not shown).

\section{mPFC frequency changes reflects spontaneous pain perception in CBP}

We next assessed the relationship between the high-frequency oscillations of the BOLD signal in the MPFC to pain perception. We test the specific hypothesis that the mPFC region identified as having higher-frequency BOLD oscillations in CBP is the same 

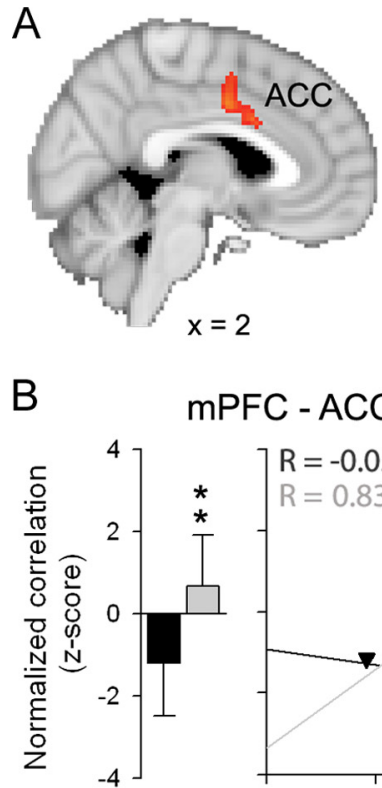

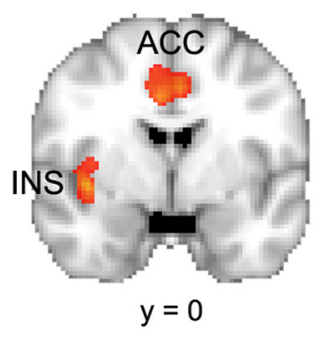

MPFC - ACC

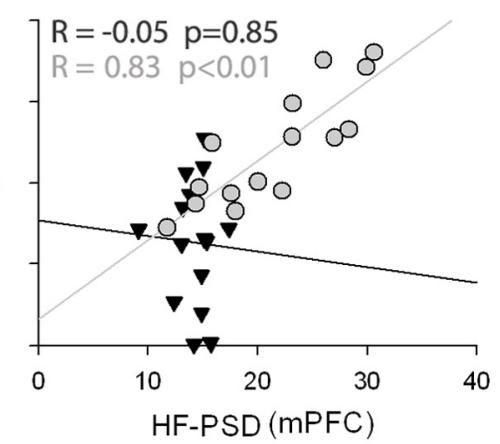

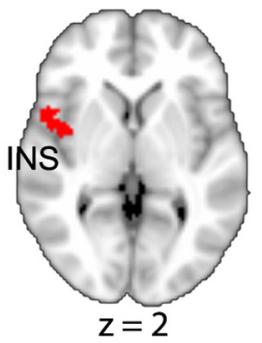

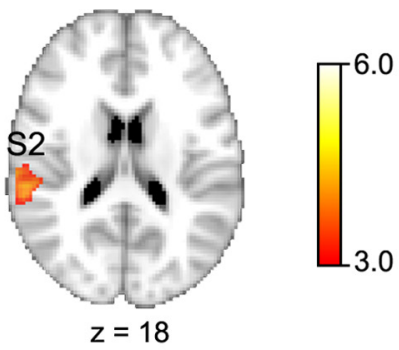

C mPFC - INS
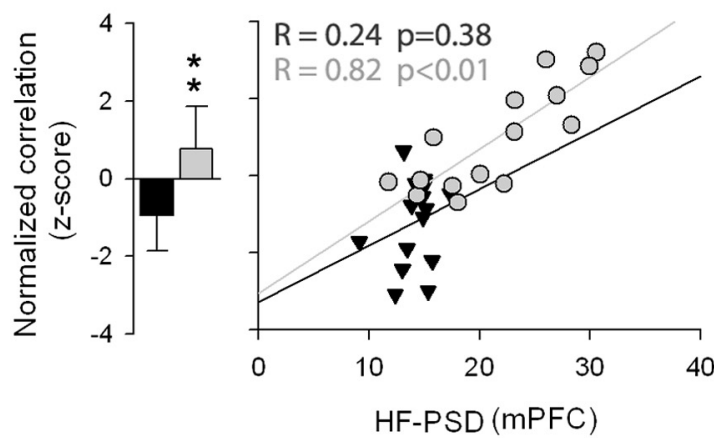

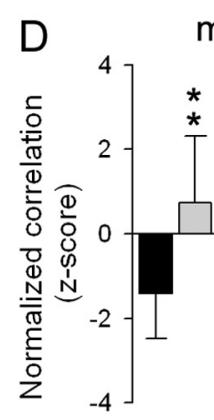

mPFC - S2
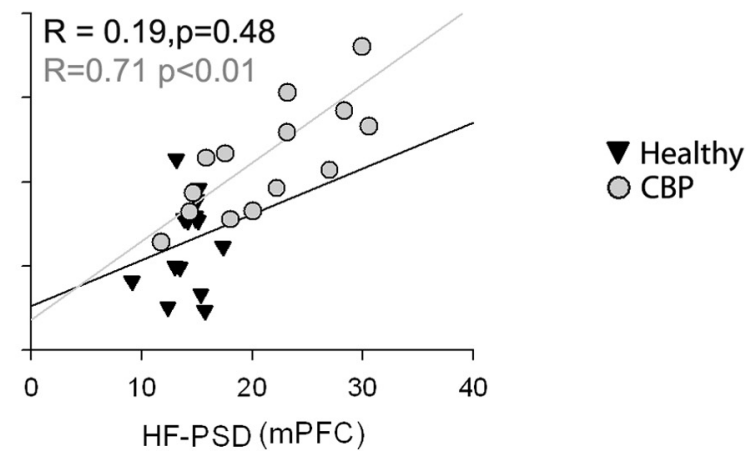

Figure 3. Functional connectivity differences for the mPFC between healthy subjects and CBP during resting-state fMRI. $\boldsymbol{A}$, Whole-brain voxelwise contrast in $\mathrm{mPFC}$ connectivity between CBP and healthy subjects. Brain regions in red-yellow depict statistically significant increased mPFC connectivity in CBP compared to controls (unpaired $t$ test, random-effects model, $Z$-score $>3.0$, cluster $p<0.01$, corrected for multiple comparisons). (BP showed increased correlation between the $\mathrm{mPFC}$ and ACC, INS, and S2, regions known to be involved in pain perception and modulation. Brain images are in standard MNI space, and coordinates are in millimeters. $B$, Bar graphs show the mean \pm SD of normalized correlation values ( $z$-scores) between $\mathrm{mPFC}$ and ACC BOLD time courses (6 $\mathrm{mm} \mathrm{ROI)} \mathrm{for} \mathrm{healthy} \mathrm{subjects} \mathrm{(black} \mathrm{bars)} \mathrm{and} \mathrm{(BP} \mathrm{(gray} \mathrm{bars).} \mathrm{The} \mathrm{mPFC}$ showed a negative correlation with the ACC in healthy subjects. This relationship was significantly reversed in CBP. Scatter plot depicts the relationship between the high-frequency spectral power (HF-PSD) for the mPFC BOLD time courses and the strength of mPFC-ACC correlations in healthy subjects (black triangles) and CBP (gray circles). The strength of the mPFC-ACC correlation showed a significant positive relationship with mPFC BOLD HF-PSD in CBP $(r=0.83, p<0.01)$ but not in healthy subjects $(r=$ $-0.05, p=0.85)$. C, D, Same as $\boldsymbol{B}$ for mPFC-INS and mPFC-S2 correlations. ${ }^{* *} p<0.01$.

area that reflects fluctuations of back pain in the task where CBP patients are actually rating their spontaneous pain (the region is in fact very close to mPFC identified in the past using a general linear modeling analysis for ratings of back pain; Baliki et al., 2006). Thus, we use the mPFC coordinates from the resting-state contrast to look for the properties of this region in relation to back pain ratings. Thirteen of the CBP subjects rated their online fluctuations of spontaneous pain for $10 \mathrm{~min}$ in the absence of external intervention during the scan (pain rating task). The individual pain ratings from the 13 CBP patients are shown in Figure $4 \mathrm{~A}$. The $\mathrm{mPFC}$ BOLD time courses during rating of spontaneous pain (6 $\mathrm{mm}$ ROI peak coordinates derived from restingstate fMRI) were computed for each individual and submitted for a spectrogram analysis using $90 \mathrm{~s}$ windows with $95 \%$ overlap. This allowed us to track the change in power for the entire BOLD frequency range in time. An example of the mPFC BOLD time course from the pain rating scan from patient 7 is shown in Figure
$4 B$. The spectrogram derived from the corresponding BOLD signal is shown in Figure $4 C$. The spectrogram was used to compute time courses for the three frequency bands by averaging the power for each time point within the given band and normalizing them to their respective means (Fig. $4 C$, bottom). To directly correlate these time courses with the pain perception, the pain rating was (1) convolved with the hemodynamic response function (to compensate for delay of blood flow), (2) resampled in time to match the brain activity time courses (Fig. 4D), and (3) submitted to a Pearson correlation analysis separately for the low, middle, and high-frequency band. The same analysis was performed for visual ratings obtained for the visual rating task, in which subjects rated online changes in the length of a bar projected on the screen.

Figure $4 E$ shows the group average correlation for pain rating and visual ratings with brain activity time courses derived for the three frequency bands. The high-frequency oscillations in the mPFC BOLD time course exhibited statistically significant posi- 
A

\section{Spontaneous pain ratings}
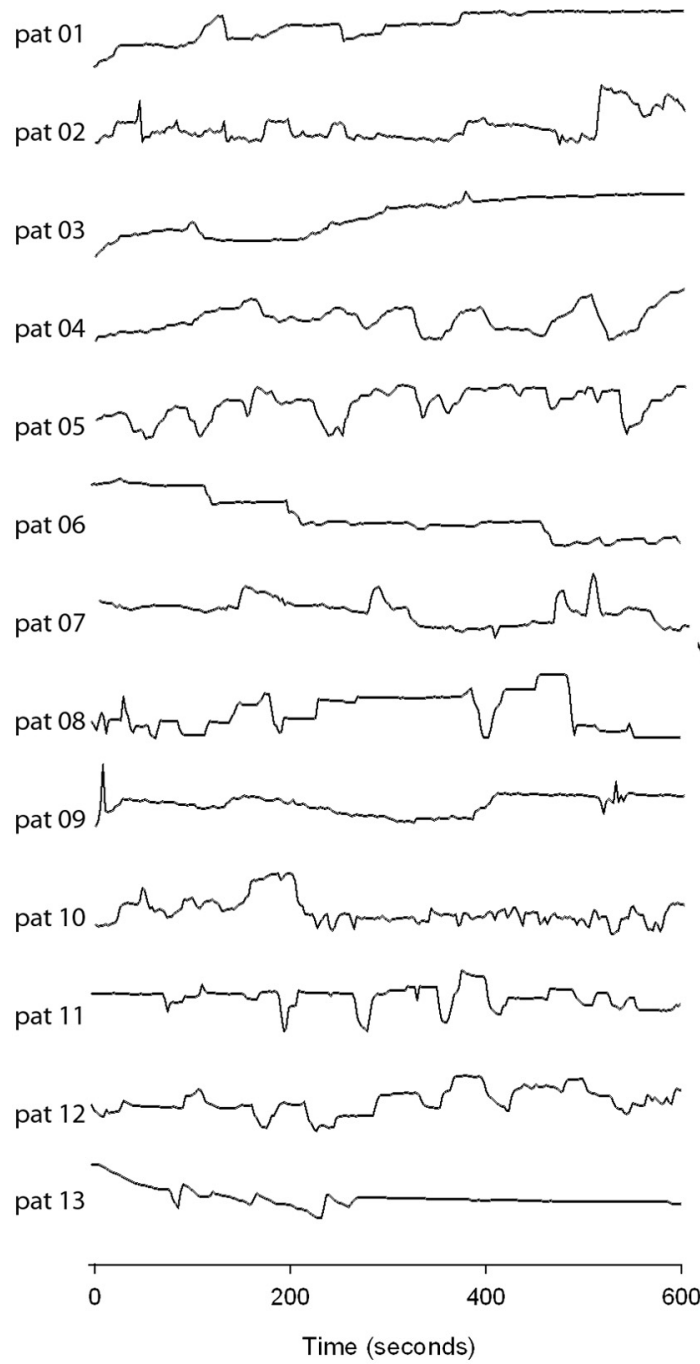

B

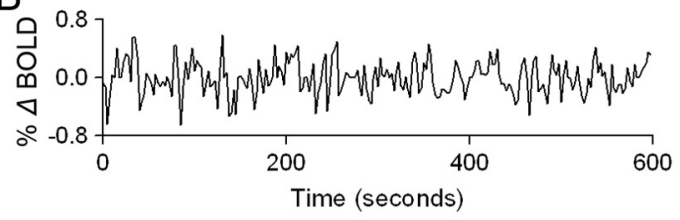

C
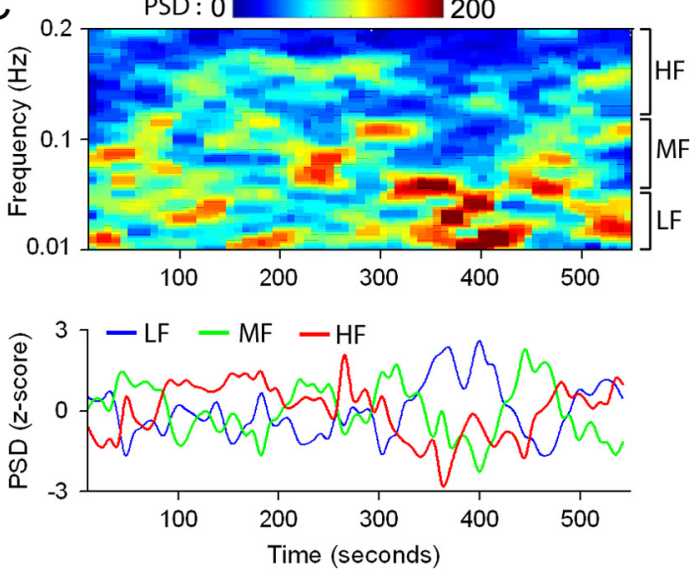

D

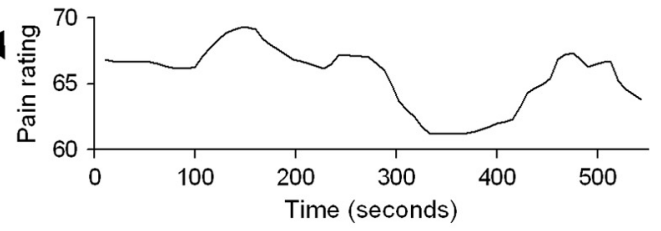

$E$

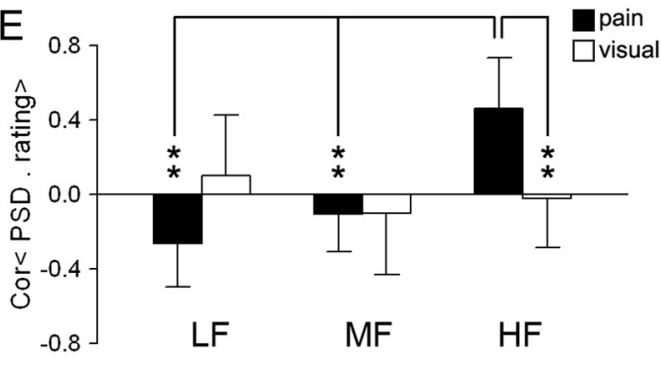

Figure 4. Relationship between mPFC BOLD spectral power and spontaneous pain ratings in CBP. A, Spontaneous pain ratings for 13 CBP patients. Patients used a finger-span device to continuously rate spontaneous fluctuations of their back pain (pain rating task) on a scale of 0 - 100 in the absence of external stimulation during fMRI scanning. In a separate scan, patients also performed a visual control task, in which they rated the size of a bar projected on a screen. $\boldsymbol{B}$, An example of the $\mathrm{mPFC} B 0 \mathrm{LD}$ time course from CBP patient 07 (pat 07) during the pain-rating task. C, Top color panel shows the spectrogram of the mPFC BOLD signal. Lower line plots show the averaged normalized spectral power for the low- (LF, $0.01-0.05 \mathrm{~Hz}$, blue trace), mid- (MF, $0.05-0.12 \mathrm{~Hz}$, green trace), and high- (HF, $0.12-0.25$, $\mathrm{Hz}$, red trace) frequency bands derived from the spectrogram. Power was averaged across all frequencies for a given band and normalized with respect to the mean. $D$, Spontaneous pain rating for patient 7 , after convolving with homodynamic response function and temporal resampling to match the time course of the spectrogram. The pain rating exhibits similar temporal fluctuations only to the high-frequency band spectrum. $E$, Bar graphs show the mean \pm SD Pearson correlation coefficients of the ratings and power spectra for the three frequency bands during pain rating (black bars) and visual rating (white bars) tasks. Individual patients' ratings showed a significant relationship only to the $\mathrm{mPFC}$ power for the high-frequency band and only for pain rating task. ${ }^{* *} p<0.01$.

tive correlations with pain rating, but not with visual ratings (pain rating: $0.46 \pm 0.28$; visual rating: $-0.03 \pm 0.35$; two-sided unpaired $t$ test: $t=5.89, p<0.01)$. In addition, neither pain nor visual ratings showed any statistically significant correlations with brain activity of the low-frequency band (pain rating: $-0.24 \pm 0.23$; visual rating: $0.09 \pm 0.32$ ) and mid-frequency band (pain rating: $-0.11 \pm 0.20$; visual rating: $-0.10 \pm 0.33$ ). These results indicate that the high-frequency events in the mPFC BOLD signal are associated with the fluctuations of spontaneous pain of CBP reported by these patients.

\section{Discussion}

The current investigation is the most recent installment of a series of studies aimed to investigate the neural mechanisms associated with and modulated by spontaneous pain in CBP patients. The first seminal observation was that spontaneous $\mathrm{CBP}$ had a unique brain representation different from that of acute pain. Elevated spontaneous CBP pain was perpetuated by sustained activation of the medial prefrontal cortex (a region involved in emotional/ self-referential information processing) and punctuated by transient nociceptive input that perpetuate the state of continued negative affect (suffering) regarding the self in CBP patients (Baliki et al., 2006). These results, coupled with behavioral data, showing impaired performance of CBP patients on a gambling task (Apkarian et al., 2004b), led us to the conclusion that chronic pain can no longer be fully characterized within the classical pain system. We reasoned that CBP might indeed impact and modu- 
late brain functions beyond the pain system itself. This spurred our second study, in which we investigated the functional reorganization of brain networks in CBP patients during the performance of a simple attention task (Baliki et al., 2008). We discovered that CBP patients, despite performing the task as well as healthy controls, displayed increased activation in several key DMN regions. This abnormal activity was also associated with disrupted correlation/anti-correlation of selective regions with the DMN and attention networks, thus demonstrating that chronic pain has a widespread impact on overall brain function. The current study is designed to further identify the cortical "signature" of CBP and explore the predominant mechanisms of CBP perception. We observe that CBP is associated with a shift toward higher frequencies in the oscillatory activity in the brains of patients during rest as measured by fMRI. These enhanced high-frequency fluctuations primarily mapped to the mPFC and brain regions within the DMN, thus corroborating our previous findings. Functionally, the shift in oscillations was associated with reorganized connectivity between the MPFC and insula, cingulate, and secondary somatosensory regions, areas involved in pain processing and receiving inputs from peripheral pain pathways. In addition to changes in function, increases in highfrequency fluctuations exhibited a significant temporal coupling with surges in spontaneous CBP intensity.

The functional relevance of high-frequency BOLD oscillations $(0.10-0.25 \mathrm{~Hz})$ is not fully understood. Multiple groups have examined resting-state fMRI for higher-frequency BOLD oscillations (Mitra et al., 1997; Cordes et al., 2002; Wise et al., 2004; Duff et al., 2008; Salvador et al., 2008; He et al., 2010; Malinen et al., 2010; Zuo et al., 2010). Recently we showed that BOLD fluctuations pertain to a spatiotemporal organization with different frequency mapping to distinct brain networks (Baria et al., 2011). In accordance to these findings, here we show that spontaneous activity in healthy subjects exhibit a frequencydependent large-scale neural organization with limbic, subcorti$\mathrm{cal}$, and temporal regions displaying largest measures of power in higher-frequency bands, while visual, parietal, and frontal regions are dominated with lowest-frequency BOLD oscillations, replicating our recent findings (Baria et al., 2011). Compared to controls, CBP patients exhibited a similar organization with a shift to high frequencies especially in the MPFC and brain regions within the DMN network, including the PCC and bilateral LP regions. These increases in high-frequency power could reflect changes in ongoing neural activity and/or cortical excitability associated with spontaneous pain, since mutually interacting oscillations would be well positioned to regulate information integration across multiple spatial and temporal scales (Canolty and Knight, 2010). Brain oscillations have been a prominent theme in animal (local field potential) and human (electroencephalography) research, with different frequency bands distinguishing specific behavioral states such as sleep, cognition, eye closure, and activation (He et al., 2010). Within this framework, there is accumulating evidence showing that changes in BOLD oscillation may also be related to changes in behavioral state such as task performance and thus may reflect changes in neural activity (Duff et al., 2008; Baria et al., 2011).

Modulation of BOLD temporal fluctuations has been observed within the resting-state networks of two other chronic pain patient groups. Cauda and colleagues observed increased power in the $0.05-0.25 \mathrm{~Hz}$ range of the DMN in patients suffering from diabetic neuropathy (Cauda et al., 2009a). Likewise, Malinen and colleagues showed that patients with chronic pain exhibited increased power in the high-frequency band in the
ACC and INS (Malinen et al., 2010). Both studies argued that these alterations reflect a change in the functional connectivity of the related brain regions involved in both affective (ACC and INS) and sensory (thalamus, S1 and S2) processing of pain perception. When we examined the intrinsic connectivity of the mPFC during rest, we observed congruent modulations. The mPFC showed localized increased functional connectivity (shift from negative to positive correlations) to the ACC, INS, and S2. These results suggest that the change in the oscillatory properties is coupled with change in functional connectivity, which can be explained as increased nociceptive input via peripheral pain pathways that permeates the brain and disturbs resting-state dynamics. Thus, the affected neuronal assemblies receiving input from nociceptive pathways turn into disorderly sources of central generators swaying the system toward inflexibility and attenuating its ability in sampling information along different discharge frequency bands (Cauda et al., 2009a). It can therefore be suggested that this "signature" of restructured connectivity is mirrored in changes of correlation/anti-correlation within the DMN network observed in our previous work (Baliki et al., 2008) and by other groups (Cauda et al., 2009a; Tagliazucchi et al., 2010).

In addition to changes in functional connectivity, the shift in the frequency profile within the $\mathrm{mPFC}$ mirrored changes in spontaneous pain intensity in CBP. This observation provides direct evidence for the specificity of the BOLD frequency changes observed in patients since it exhibits direct correlation to their pain report in real time. Furthermore, this correlation was unique for changes in spontaneous pain, since it was not observed when subjects rated the length of a moving bar (visual control task). These results are consistent with our previous observations that the activity in the mPFC is sustained during high-pain periods in patients using a general linear model (GLM) analysis (Baliki et al., 2006). Thus, using two different approaches (frequency-based analysis in resting-state fMRI and GLM analysis during pain rating), we were able to identify the $\mathrm{mPFC}$ as the primary module underlying spontaneous pain processing in CBP. In a previous study, we also showed that mPFC activity is mediated by more transient bursts in activity in the ACC and INS (Baliki et al., 2006). This mPFC-ACC and mPFC-Ins coupling is also evident in this study in the form of increased correlation within these regions that is directly dependent on increases in high-frequency power in the mPFC. It is worthy to note that complementary results were reported by Tagliazucchi and colleagues, where they showed that brain regions within the frontal cortex, mainly orbital areas, show increased coactivation with bilateral insula in CBP patients during resting-state fMRI (Tagliazucchi et al., 2010). The neuronal mechanisms linking spontaneous pain processing to high-frequency BOLD fluctuations within the frontal cortex remains unknown and can be attributed to both peripheral and central processes. Peripherally, it can be due to increased ectopic discharges in nociceptive fibers coupled with enhanced spinal prefrontal projections through the spinothalamic tract and other pathways such as spinoparabrachial, spinostriatal, and spinoreticular pathways (Willis and Westlund, 1997; Gauriau and Bernard, 2002; Braz et al., 2005). Alternatively, the observed increase in high-frequency oscillations can be the result of central changes, including increased disinhibition of thalamocortical projections (Cauda et al., 2009a) and/or morphological changes, which have been shown in CBP patients (Apkarian et al., 2004a) and other chronic pain conditions (Davis et al., 1995; SchmidtWilcke et al., 2005; Schmidt-Wilcke et al., 2007; Seminowicz and Davis, 2007; Kim et al., 2008; May, 2008; Valfrè et al., 2008; Blankstein et al., 2010). 
This is the first study that directly links temporal changes in high-frequency oscillations to a behavioral output, linking perception and high-frequency BOLD. Recent studies indicate that BOLD signal reflects local dendrosomatic processing (Lippert et al., 2010) and is due mainly to excitatory neuronal activity (Lee et al., 2010), suggesting that the changes in high-frequency fluctuation observed in this study are a consequence of altered neuronal firing properties. However, it has been shown that cardiac and respiratory rhythms as well as head motion artifacts are aliased into the high-frequency spectrum of the BOLD signal (De Luca et al., 2006; Duff et al., 2008). Here increases in high power are unlikely due to physiological or motion artifacts, since the spatial distribution of regions showing increased high-frequency fluctuations did not map to regions and/or edges typically associated with physiological noise or motion artifacts. Furthermore, the two groups showed minimal head motion (healthy: $1.12 \pm 1.89 \mathrm{~mm}$; CBP: $1.69 \pm 1.44 \mathrm{~mm}$; two-sided unpaired $t$ test: $t=-0.76, p=0.87$ ), and regression of motion parameters from the brain data did not affect the observed changes in the power distribution.

In conclusion, these findings provide novel insights about the nature of $\mathrm{CBP}$ and the mechanisms by which it alters brain function. Spontaneous pain in chronic pain seems uniquely conveyed through increased high-frequency oscillations during rating of ongoing pain, and the same brain region in resting brain activity in turn also reflects changes in connectivity within resting-state networks, especially between the frontal cortex and pain related regions. It is our contention that these changes, coupled with changes in brain salience and motivation valuation circuitry, are essential mediators for the functional, cognitive, and behavioral alterations commonly seen in chronic pain patients.

\section{References}

Apkarian AV, Sosa Y, Sonty S, Levy RM, Harden RN, Parrish TB, Gitelman DR (2004a) Chronic back pain is associated with decreased prefrontal and thalamic gray matter density. J Neurosci 24:10410-10415.

Apkarian AV, Sosa Y, Krauss BR, Thomas PS, Fredrickson BE, Levy RE, Harden RN, Chialvo DR (2004b) Chronic pain patients are impaired on an emotional decision-making task. Pain 108:129-136.

Apkarian AV, Bushnell MC, Treede RD, Zubieta JK (2005) Human brain mechanisms of pain perception and regulation in health and disease. Eur J Pain 9:463-484.

Apkarian AV, Hashmi JA, Baliki MN (2011) Pain and the brain: specificity and plasticity of the brain in clinical chronic pain. Pain 152:S49-64.

Baliki MN, Chialvo DR, Geha PY, Levy RM, Harden RN, Parrish TB, Apkarian AV (2006) Chronic pain and the emotional brain: specific brain activity associated with spontaneous fluctuations of intensity of chronic back pain. J Neurosci 26:12165-12173.

Baliki MN, Geha PY, Apkarian AV, Chialvo DR (2008) Beyond feeling: chronic pain hurts the brain, disrupting the default-mode network dynamics. J Neurosci 28:1398-1403.

Baria AT, Baliki MN, Parrish T, Apkarian AV (2011) Anatomical and functional assemblies of brain BOLD oscillations. J Neurosci 31:7910-7919.

Beck AT, Steer RA (1993) Manual for the Beck Anxiety Inventory. San Antonio: Psychological Corporation.

Blankstein U, Chen J, Diamant NE, Davis KD (2010) Altered brain structure in irritable bowel syndrome: potential contributions of pre-existing and disease-driven factors. Gastroenterology 138:1783-1789.

Braz JM, Nassar MA, Wood JN, Basbaum AI (2005) Parallel "pain” pathways arise from subpopulations of primary afferent nociceptor. Neuron 47:787-793.

Canolty RT, Knight RT (2010) The functional role of cross-frequency coupling. Trends Cogn Sci 14:506-515.

Cauda F, Sacco K, Duca S, Cocito D, D'Agata F, Geminiani GC, Canavero S (2009a) Altered resting state in diabetic neuropathic pain. PLoS One $4: e 4542$.

Cauda F, Sacco K, D’Agata F, Duca S, Cocito D, Geminiani G, Migliorati F, Isoardo G (2009b) Low-frequency BOLD fluctuations demonstrate al- tered thalamocortical connectivity in diabetic neuropathic pain. BMC Neurosci 10:138.

Cauda F, D'Agata F, Sacco K, Duca S, Cocito D, Paolasso I, Isoardo G, Geminiani G (2010) Altered resting state attentional networks in diabetic neuropathic pain. J Neurol Neurosurg Psychiatry 81:806-811.

Cordes D, Haughton V, Carew JD, Arfanakis K, Maravilla K (2002) Hierarchical clustering to measure connectivity in fMRI resting-state data. Magn Reson Imaging 20:305-317.

Davis KD, Wood ML, Crawley AP, Mikulis DJ (1995) fMRI of human somatosensory and cingulate cortex during painful electrical nerve stimulation. Neuroreport 7:321-325.

De Luca M, Beckmann CF, De Stefano N, Matthews PM, Smith SM (2006) fMRI resting state networks define distinct modes of long-distance interactions in the human brain. Neuroimage 29:1359-1367.

Duff EP, Johnston LA, Xiong J, Fox PT, Mareels I, Egan GF (2008) The power of spectral density analysis for mapping endogenous BOLD signal fluctuations. Hum Brain Mapp 29:778-790.

Fox MD, Snyder AZ, Vincent JL, Corbetta M, Van Essen DC, Raichle ME (2005) The human brain is intrinsically organized into dynamic, anticorrelated functional networks. Proc Natl Acad Sci U S A 102:9673-9678.

Fukunaga M, Horovitz SG, van Gelderen P, de Zwart JA, Jansma JM, Ikonomidou VN, Chu R, Deckers RH, Leopold DA, Duyn JH (2006) Largeamplitude, spatially correlated fluctuations in BOLD fMRI signals during extended rest and early sleep stages. Magn Reson Imaging 24:979-992.

Gauriau C, Bernard JF (2002) Pain pathways and parabrachial circuits in the rat. Exp Physiol 87:251-258.

Harden RN, Weinland SR, Remble TA, Houle TT, Colio S, Steedman S, Kee WG (2005) Medication Quantification Scale Version III: update in medication classes and revised detriment weights by survey of American Pain Society physicians. J Pain 6:364-371.

He BJ, Zempel JM, Snyder AZ, Raichle ME (2010) The temporal structures and functional significance of scale-free brain activity. Neuron 66:353-369.

Jenkins GM, Watts DG (1968) Spectral analysis and its applications. Boca Raton, FL: Emerson-Adams.

Jenkinson M, Smith S (2001) A global optimisation method for robust affine registration of brain images. Med Image Anal 5:143-156.

Kim JH, Suh SI, Seol HY, Oh K, Seo WK, Yu SW, Park KW, Koh SB (2008) Regional grey matter changes in patients with migraine: a voxel-based morphometry study. Cephalalgia 28:598-604.

Lee JH, Durand R, Gradinaru V, Zhang F, Goshen I, Kim DS, Fenno LE, Ramakrishnan C, Deisseroth K (2010) Global and local fMRI signals driven by neurons defined optogenetically by type and wiring. Nature 465:788-792.

Lippert MT, Steudel T, Ohl F, Logothetis NK, Kayser C (2010) Coupling of neural activity and $\mathrm{AMRI}-\mathrm{BOLD}$ in the motion area MT. Magn Reson Imaging 28:1087-1094.

Malinen S, Vartiainen N, Hlushchuk Y, Koskinen M, Ramkumar P, Forss N, Kalso E, Hari R (2010) Aberrant temporal and spatial brain activity during rest in patients with chronic pain. Proc Natl Acad Sci U S A 107:6493-6497.

May A (2008) Chronic pain may change the structure of the brain. Pain 137:7-15.

Melzack R (1987) The short-form McGill Pain Questionnaire. Pain 30:191-197.

Mitra PP, Ogawa S, Hu X, Uğurbil K (1997) The nature of spatiotemporal changes in cerebral hemodynamics as manifested in functional magnetic resonance imaging. Magn Reson Med 37:511-518.

Napadow V, LaCount L, Park K, As-Sanie S, Clauw DJ, Harris RE (2010) Intrinsic brain connectivity in fibromyalgia is associated with chronic pain intensity. Arthritis Rheum 62:2545-2555.

Price DD (2000) Psychological and neural mechanisms of the affective dimension of pain. Science 288:1769-1772.

Salvador R, Martínez A, Pomarol-Clotet E, Gomar J, Vila F, Sarró S, Capdevila A, Bullmore E (2008) A simple view of the brain through a frequencyspecific functional connectivity measure. Neuroimage 39:279-289.

Schmidt-Wilcke T, Leinisch E, Straube A, Kämpfe N, Draganski B, Diener HC, Bogdahn U, May A (2005) Gray matter decrease in patients with chronic tension type headache. Neurology 65:1483-1486.

Schmidt-Wilcke T, Luerding R, Weigand T, Jurgens T, Schuierer G, Leinisch E, Bogdahn U (2007) Striatal grey matter increase in patients suffering from fibromyalgia - a voxel-based morphometry study. Pain 132 [Suppl 1]:S109-S116.

Seminowicz DA, Davis KD (2007) Pain enhances functional connectivity of 
a brain network evoked by performance of a cognitive task. J Neurophysiol 97:3651-3659.

Smith SM, Jenkinson M, Woolrich MW, Beckmann CF, Behrens TE, Johansen-Berg H, Bannister P, De Luca CJ, Drobnjak I, Flitney DE, Nianzy R, Saunders J, Vickers J, Zhang Y, De Stefano N, Brady JM, Mathews PM (2004) Advances in functional and structural MR image analysis and implementation as FSL. Neuroimage 23 [Suppl 1]:208-219.

Tagliazucchi E, Balenzuela P, Fraiman D, Chialvo DR (2010) Brain resting state is disrupted in chronic back pain patients. Neurosci Lett 485:26-31.

Valfrè W, Rainero I, Bergui M, Pinessi L (2008) Voxel-based morphometry reveals gray matter abnormalities in migraine. Headache 48:109-117.

Van Dijk KR, Hedden T, Venkataraman A, Evans KC, Lazar SW, Buckner
RL (2010) Intrinsic functional connectivity as a tool for human connectomics: theory, properties, and optimization. J Neurophysiol 103: 297-321.

Van Essen DC (2005) A population-average, landmark- and surface-based (PALS) atlas of human cerebral cortex. NeuroImage 28:635-662.

Willis WD, Westlund KN (1997) Neuroanatomy of the pain system and of the pathways that modulate pain. J Clin Neurophysiol 14:2-31.

Wise RG, Ide K, Poulin MJ, Tracey I (2004) Resting fluctuations in arterial carbon dioxide induce significant low frequency variations in BOLD signal. Neuroimage 21:1652-1664.

Zuo XN, Di Martino A, Kelly C, Shehzad ZE, Gee DG, Klein DF, Castellanos FX, Biswal BB, Milham MP (2010) The oscillating brain: complex and reliable. Neuroimage 49:1432-1445. 